\title{
Genetic modification of the effects of exercise behavior on mental health
}

\author{
Nienke M. Schutte ${ }^{1,2}$, Meike Bartels ${ }^{1,2}$ and Eco J. C. de Geus ${ }^{1,2}$ \\ ${ }^{1}$ Department of Biological Psychology, Faculty of Psychology and Education, VU University Amsterdam, Amsterdam, Netherlands \\ ${ }^{2}$ EMGO+ Institute for Health and Care Research, VU University Medical Center Amsterdam, Amsterdam, Netherlands \\ *Correspondence: n.m.schutte@vu.nl \\ Edited by: \\ Felipe Schuch, Hospital de Clinicas de Porto Alegre, Brazil \\ Reviewed by: \\ Eduardo Lusa Cadore, Federal University of Rio Grande do Sul, Brazil
}

Keywords: exercise, depression, wellbeing, mood effects, individual differences, genetics

Anxiety and depressive disorders are a major contributor to the global disease burden (1). Although these disorders differ in duration and intensity, they are often chronic and treatment options include medication, psychotherapy, or a combination of both. In addition, regular exercise is argued to be effective in reducing anxious and depressive symptoms. Results from several meta-analyses indicate that exercise has a moderate to large antidepressant effect in clinical populations (2-6). Based on these studies, one might easily conclude that exercise consistently has beneficial causal effects on anxious and depressive symptoms (7).

The question remains whether this conclusion is also valid with regard to the general population as, despite these beneficial psychological effects, the majority of the population is not engaging in leisuretime exercise activities $(8,9)$ and population studies on the association between exercise and mental health are scarce. Secondly, there may be mechanisms that only mimic causal effects. The observed association between exercise and anxiousdepressive symptoms might be due to underlying factors that influence both exercise behavior and symptoms of anxiety and depression. These factors can reside in the environment or in our genes. Underlying genetic factors might for instance have a detrimental effect on regular exercise behavior while simultaneously increasing the risk for depression, a mechanism known as genetic pleiotropy. The effect of these genetic factors on exercise behavior could even precede their effects on depression, thereby nearly perfectly mimicking a causal association. Only a few research groups have the optimal resources to investigate these possible effects in a genetically informative design, which requires large population-based longitudinal datasets with family data, but preferably twin data.

Results from population-based twin studies that have tested the nature of the association between a lack of exercise and anxious-depressive symptoms conclude that the association is best explained by underlying genetic effects. De Moor et al. (10) showed that within genetically identical twins, a twin who exercised more did not have fewer symptoms than his or her less exercising co-twin. This suggests that genetic factors independently cause low levels of exercise behavior as well as anxious and depressive symptoms. In addition, there is no evidence for causal influences of exercise behavior on feelings of psychological wellbeing, a phenotype presumably at the other end of the emotional scale, i.e., the absence of anxious or depressive symptoms $(11,12)$. Taken together, these studies conclude that the association between regular exercise and psychological wellbeing as well as the association between a lack of regular exercise and anxiety and depressive disorders largely reflect the effects of common genetic factors.

In an effort to explain the mechanisms that contribute to the association between exercise activities and mental health in the general population, a model was proposed that accommodates genetic pleiotropic effects, but still allows exercise to causally increase wellbeing in specific subgroups of the population (13). As with any other behavior, for exercise behavior to be repeated regularly, the net appetitive effects of exercise would need to outweigh the net aversive effects. Individuals who experience greater exercise induced mood enhancement are likely to repeat the behavior and become regular lifetime exercisers. This assumption is supported by several studies, which show that a more positive affective response during exercise was associated with greater participation in (voluntary) moderate to vigorous exercise $(14,15)$ or the intention to engage in voluntary exercise (16). Individual differences in these acute mood effects of exercise could be strongly co-determined by genetic factors.

In addition to differential acute mood effects, there could be a socialpsychological mechanism that makes some individuals more attracted to exercise than others. Individuals with higher innate exercise capacities will gain more in exercise performance than others at comparable levels of training. The higher trainability and the superior exercise performance will lead to feelings of competence and mastery. This increased confidence, or self-efficacy, may not only enhance the frequency of exercise in individuals (17), but will also lead to higher self-esteem and in turn, in feelings of wellbeing. Vice versa, low trainability and lower levels of performance will lead to disappointment and particularly in adolescents to shame and lowered self-esteem. Genetic variation among people influencing exercise ability will therefore become associated with experiencing psychological beneficial effects of exercise activities and, as a consequence, with an increase in the frequency of exercising. 
Major future challenges are to test the association between the level of voluntary exercise behavior and the acute and longer term psychological responses to exercise, and to establish the contribution of shared genetic factors to these associations. This requires a substantial family or twin study with measurements of exercise ability and the acute mood response to exercise. Various experimental design issues should be taken into account in these studies. First, the intensity at which an individual is exercising is an important determinant of the aversive responses to exercise: at intensities that exceed the individuals' ventilatory threshold (VT), when there is a transition from aerobic to anaerobic metabolism, negative changes in exercise induced mood response are observed (18). Measurements should therefore be standardized for the VT. Second, different types of exercise induced mood responses can be measured: during (immediate response on exercising) or (shortly) after the exercise bout (more complex, long lasting feelings). These responses may differ in origin, but are likely to contribute to the overall balance of appetitive and aversive effects of exercise, therefore, should both be included in measurements. For the assessment of exercise ability it is important to take into account a range of objective determinants like aerobic fitness, balance, flexibility and static, and dynamic muscle strength, but also record self-perceived exercise ability, particularly in relation to the relevant peer group.

Acknowledgment of the differential sensitivity to the psychological effects of exercise is of great importance. Some individuals may require a specific exercise program (with respect to intensity of exercise, absence or presence of competitive elements, and type of exercise) to create a situation in which the appetitive effects of exercise can predominate. This may ensure that these individuals continue to be engaged in regular exercise while maximizing their psychological benefits in terms of increased feelings of wellbeing and decreased levels of anxiety and depression.

\section{REFERENCES}

1. Ferrari AJ, Charlson FJ, Norman RE, Patten SB, Freedman G, Murray CJL, et al. Burden of depressive disorders by country, sex, age, and year: findings from the global burden of disease study 2010. PLoS Med (2013) 10:e1001547. doi:10.1371/ journal.pmed.1001547

2. Craft LL, Landers DM. The effect of exercise on clinical depression and depression resulting from mental illness: a meta-analysis. J Sport Exerc Psychol (1998) 20:339-57.

3. Lawlor DA, Hopker SW. The effectiveness of exercise as an intervention in the management of depression: systematic review and metaregression analysis of randomized controlled trials. BMJ (2001) 322:763-7. doi:10.1136/bmj.322. 7289.763

4. Stathopoulou G, Powers MB, Berry AC, Smits JAJ, Otto MW. Exercise interventions for mental health: a quantitative and qualitative review. Clin Psychol Sci Pract (2006) 13:179-93. doi:10.1111/j.14682850.2006.00021.x

5. Krogh J, Nordentoft M, Sterne JA, Lawlor DA. The effect of exercise in clinically depressed adults: systematic review and meta-analysis of randomized controlled trials. J Clin Psychiatry (2011) 72:529-38. doi:10.4088/JCP.08r04913blu

6. Josefsson T, Lindwall M, Archer T. Physical exercise intervention in depressive disorders: metaanalysis and systematic review. Scand J Med Sci Sports (2014) 24:259-72. doi:10.1111/sms.12050

7. Stanton R, Happell B, Hayman M, Reaburn P. Exercise interventions for the treatment of affective disorders - research to practice. Front Psychiatry (2014) 5:46. doi:10.3389/fpsyt.2014.00046

8. Martínez-González MA, Varo JJ, Santos JL, de Irala J, Gibney M, Kearney J, et al. Prevalence of physical activity during leisure time in the European Union. Med Sci Sports Exerc (2001) 33:1142-6. doi:10.1097/00005768-200107000-00011

9. Troiano RP, Berrigan D, Dodd KW, Mâsse LC, Tilert T, McDowell M. Physical activity in the United States measured by accelerometer. Med Sci Sports Exerc (2008) 40:181-8. doi:10.1249/mss. 0b013e31815a51b3

10. De Moor MHM, Boomsma DI, Stubbe JH, Willemsen G, de Geus EJC. Testing causality in the association between regular exercise and symptoms of anxiety and depression. Arch Gen Psychiatry (2008) 65(8):879-905. doi:10.1001/archpsyc.65.8.897

11. Stubbe JH, de Moor MHM, Boomsma DI, de Geus EJC. The association between exercise participation and wellbeing: a co-twin study. Prev
Med (2007) 44:148-52. doi:10.1016/j.ypmed.2006. 09.002

12. Bartels M, de Moor MHM, van der Aa N, Boomsma DI, de Geus EJC. Regular exercise, subjective wellbeing, and internalizing problems in adolescence: causality or genetic pleiotropy? Front Genet (2012) 3:4. doi:10.3389/fgene.2012.00001

13. De Geus EJC, de Moor MHM. A genetic perspective on the association between exercise and mental health. Ment Health Phys Act (2008) 1:53-61. doi:10.1016/j.mhpa.2008.09.005

14. Schneider ML, Graham DJ. Personality, physical fitness, and affective response to exercise among adolescents. Med Sci Sports Exerc (2009) 41:947-55. doi:10.1249/MSS.0b013e31818de009

15. Williams DM, Dunsiger S, Ciccolo JT, Lewis BA, Albrecht AE, Marcus BH. Acute affective response to a moderate-intensity exercise stimulus predicts physical activity participation 6 and 12 months later. Psychol Sport Exerc (2008) 9:231-45. doi:10. 1016/j.psychsport.2007.04.002

16. Kwan BM, Bryan A. In-task and post-task affective response to exercise: translating exercise intentions into behaviour. Br J Health Psychol (2010) 15:115-31. doi:10.1348/135910709X433267

17. Dishman RK. Determinants of participation in physical activity. In: Bouchard C, Shephard RJ, Stephens T, Sutton JR, McPherson BD, editors. Exercise, Fitness and Health: A Consensus of Current Knowledge. Champaign, IL: Human Kinetics (1990). p. 75-101.

18. Ekkekakis P. Pleasure and displeasure from the body: perspectives from exercise. Cogn Emot (2003) 17:213-39. doi:10.1080/02699930302292

Conflict of Interest Statement: The authors declare that the research was conducted in the absence of any commercial or financial relationships that could be construed as a potential conflict of interest.

Received: 14 May 2014; accepted: 20 May 2014; published online: 03 June 2014.

Citation: Schutte NM, Bartels $M$ and de Geus EJC (2014) Genetic modification of the effects of exercise behavior on mental health. Front. Psychiatry 5:64. doi: 10.3389/fpsyt.2014.00064

This article was submitted to Affective Disorders and Psychosomatic Research, a section of the journal Frontiers in Psychiatry.

Copyright (ㅇ 2014 Schutte, Bartels and de Geus. This is an open-access article distributed under the terms of the Creative Commons Attribution License (CC BY). The use, distribution or reproduction in other forums is permitted, provided the original author(s) or licensor are credited and that the original publication in this journal is cited, in accordance with accepted academic practice. No use, distribution or reproduction is permitted which does not comply with these terms. 